\title{
Real-space Mapping of Surface Trap States in CIGSe Nanocrystals using 4D Electron Microscopy
}

Riya Bose, Ashok Bera, Manas R Parida, Aniruddha Adhikari, Basamat S. Shaheen, Erkki Alarousu, Jingya Sun, Tom Wu, Osman M. Bakr, and Omar F. Mohammed

Nano Lett., Just Accepted Manuscript • DOI: 10.1021/acs.nanolett.6b01553 • Publication Date (Web): 26 May 2016

Downloaded from http://pubs.acs.org on May 31, 2016

\section{Just Accepted}

"Just Accepted" manuscripts have been peer-reviewed and accepted for publication. They are posted online prior to technical editing, formatting for publication and author proofing. The American Chemical Society provides "Just Accepted" as a free service to the research community to expedite the dissemination of scientific material as soon as possible after acceptance. "Just Accepted" manuscripts appear in full in PDF format accompanied by an HTML abstract. "Just Accepted" manuscripts have been fully peer reviewed, but should not be considered the official version of record. They are accessible to all readers and citable by the Digital Object Identifier (DOI®). "Just Accepted" is an optional service offered to authors. Therefore, the "Just Accepted" Web site may not include all articles that will be published in the journal. After a manuscript is technically edited and formatted, it will be removed from the "Just Accepted" Web site and published as an ASAP article. Note that technical editing may introduce minor changes to the manuscript text and/or graphics which could affect content, and all legal disclaimers and ethical guidelines that apply to the journal pertain. ACS cannot be held responsible for errors or consequences arising from the use of information contained in these "Just Accepted" manuscripts. 


\title{
Real-space Mapping of Surface Trap States in CIGSe Nanocrystals using 4D Electron Microscopy
}

\author{
Riya Bose,$^{\dagger}$ Ashok Bera,$^{*}$ Manas R. Parida,$^{\dagger}$ Aniruddha Adhikari,$^{\dagger}$ Basamat S. Shaheen,$^{\dagger}$ Erkki \\ Alarousu, ${ }^{\dagger}$ Jingya Sun, ${ }^{\dagger}$ Tom Wu, ${ }^{\ddagger}$ Osman M. Bakr, ${ }^{\dagger}$ Omar F. Mohammed ${ }^{\dagger *}$ \\ ${ }^{\dagger}$ Solar and Photovoltaics Engineering Research Center, Physical Sciences and Engineering \\ Division, King Abdullah University of Science and Technology, Thuwal 23955-6900, Kingdom \\ of Saudi Arabia \\ \$Physical Sciences and Engineering Division, King Abdullah University of Science and \\ Technology, Thuwal 23955-6900, Kingdom of Saudi Arabia
}

\begin{abstract}
Surface trap states in semiconductor copper indium gallium selenide nanocrystals (NCs) which serve as undesirable channels for non-radiative carrier recombination, remain a great challenge impeding the development of solar and optoelectronics devices based on these NCs. In order to design efficient passivation techniques to minimize these trap states, a precise knowledge about the charge carrier dynamics on the NCs surface is essential. However, selective mapping of surface traps requires capabilities beyond the reach of conventional laser spectroscopy and static electron microscopy; it can only be accessed by using a one-of-a-kind, second-generation four-dimensional scanning ultrafast electron microscope (4D S-UEM) with sub-picosecond temporal and nanometer spatial resolutions. Here, we precisely map the
\end{abstract}


collective surface charge carrier dynamics of copper indium gallium selenide NCs as a function of the surface trap states before and after surface passivation in real space and time using SUEM. The time-resolved snapshots clearly demonstrate that the density of the trap states is significantly reduced after zinc sulfide $(\mathrm{ZnS})$ shelling. Furthermore, removal of trap states and elongation of carrier lifetime are confirmed by the increased photocurrent of the self-biased photodetector fabricated using the shelled NCs.

KEYWORDS. 4D scanning ultrafast electron microscopy, surface traps, charge carrier dynamics, CIGSe, semiconductor nanocrystals, shelling 
With their manifold tunable properties and cost-effective versatile chemical processability, research in colloidal semiconductor nanocrystals (NCs) have spanned from vast materials systems to their widespread applications in light emitting diodes, sensors and light harvesters. ${ }^{1-10}$ However, due to high surface-to-volume ratio of these NCs, the large number of unpassivated atoms on their surfaces lead to the formation of highly dense trap states, which serve as undesirable, non-radiative deactivation channels for photo-generated charge carriers. ${ }^{11-15}$ This often acts as a bottleneck in the use of these NCs for photoactive applications. Typically, multinary metal chalcogenides possess many desirable attributes, such as high absorption coefficient and high photo-stability for optoelectronic applications, ${ }^{16-21}$ and a record power conversion efficiency of $21.7 \%$ has been obtained in the bulk form following physical deposition techniques, ${ }^{22}$ which is close to the efficiency of polycrystalline silicon solar cells. But, when it comes to nanoscale, the presence of multiple trap states and the lack of the knowledge about their ultrafast carrier trapping dynamics limit their efficiency in device-based applications. ${ }^{16,23-26}$ Various surface passivation techniques, e.g., treatment with different ligands or shelling with higher bandgap materials, have been tried to reduce the detrimental effects of surface-related traps. $^{24,}$ 27-35 However, in order to design more efficient passivation strategies for photoactive materials for any applications, precise knowledge about the surface charge carrier dynamics is a prerequisite.

Ultrafast pump-probe spectroscopic techniques have been the most commonly used method to monitor the presence of carrier trapping channels in the NCs in real time. ${ }^{36-39}$ Recently, ultrafast transient absorption microscopy techniques have also been developed for spatial and temporal visualization of charge carrier dynamics in semiconductor materials. ${ }^{40-42}$ However, in these cases, the relatively large penetration depth of the laser beam allows dynamical information to be 
obtained mainly from the bulk of the sample. Therefore, selective mapping of ultrafast dynamical processes on the surfaces of semiconductor NCs remains a key challenge, and out of reach of purely optical time-resolved probing techniques. A new era of spatiotemporal imaging started with the invention of four dimensional (4D) ultrafast electron microscopy, which merged unprecedented spatial resolution with the time-resolved technique. ${ }^{43-55}$ In particular, the invention of scanning ultrafast electron microscopy (S-UEM) provided the unique opportunity to selectively map charge carrier dynamics on the surface of a material with nm spatial and subpicosecond temporal resolutions. ${ }^{56-62}$ In S-UEM, the optical pulse generated from a femtosecond (fs) laser system is used to generate electron packets from the tip of the scanning electron microscope, instead of the continuous electron beam used in the conventional setup. This pulse is synchronized with another optical excitation pulse that initiates carrier dynamics in the sample (Scheme 1). The principle of S-UEM is to detect the secondary electrons (SEs) generated in the sample, which having the most probable energy range of $2-5 \mathrm{eV}$ and are emitted from the first few nanometers of the top surface. ${ }^{63}$ Constructed at different time delays between the optical and electron pulses, these SE images give direct and precise information about the carrier dynamics on the surface of the material of interest.

Here, employing 4D S-UEM, we report real-space visualization of carrier dynamics on the surface of copper indium gallium selenide $\mathrm{NCs}\left(\mathrm{Cu}_{0.96} \mathrm{In}_{0.56} \mathrm{Ga}_{0.48} \mathrm{Se}_{2}\right.$; henceforth referred to as CIGSe) before and after surface passivation by ZnS shelling. The time-resolved SE images and the extracted kinetics obtained for CIGSe clearly reveal slower carrier recombination on the surface after shelling, thus providing direct visual evidence of the effectiveness of surface passivation. Conventional ultrafast pump-probe spectroscopic techniques also show that the overall carrier dynamics on CIGSe NCs is elongated after shelling, supporting the time-resolved 
imaging results. Moreover, an immediate effect of trap state removal can be observed in the photoresponse of the NCs. A photo-responsivity of $0.25 \mathrm{~A} / \mathrm{W}$ at $510 \mathrm{~nm}$ wavelength is obtained from the self-biased photodetector fabricated using CIGSe-ZnS NCs. This value is almost three times greater than the value obtained for the device composed of NCs before shelling. The transient photocurrent spectra also confirm significant reduction in trap states after ZnS shelling.

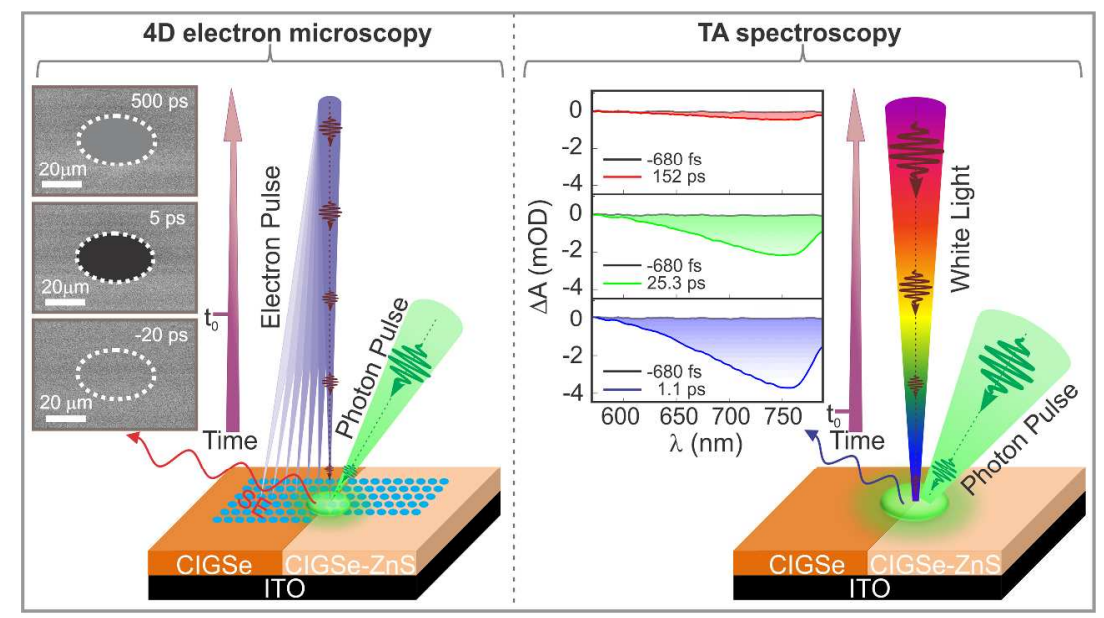

Scheme 1. Principle of S-UEM imaging (left panel) and ultrafast pump-probe spectroscopic measurements (right panel).

CIGSe nanocrystals are synthesized following high temperature precursor injection technique. The cation and anion precursors are prepared separately to ensure complete dissolution. In a typical procedure, cation precursors are dissolved separately in amine and then mixed altogether with thiol to get the thiolate complex of the precursors. ${ }^{64-65}$ A highly reactive Se precursor by dissolving selenium in 1:1 mixture of amine and thiol is used instead of the typically used selenium-oleylamine mixture. ${ }^{66}$ For $\mathrm{ZnS}$ shelling, a modified literature method was followed. ${ }^{24}$ Details of the synthesis procedure are provided in the Experimental Section. 
Figure 1(a) shows the steady-state absorption and emission spectra of CIGSe NCs before and after ZnS shelling. Absence of any clear peak in the absorption spectrum and broadness of the emission peak in these multinary NCs respectively arise due to the presence of inherent intrabandgap defect states and the radiative recombination taking place via these internal defect states. $^{23,67-68}$ This has been commonly referred as donor-acceptor pair recombination in the literature. $^{23,67-68}$ The weak intensity of emission for CIGSe NCs suggests the presence of an abundance of non-radiative channels presumably due to presence of surface states. After $\mathrm{ZnS}$ shelling, though no change is observed in the absorption spectrum, a noticeable increase in the emission intensity is observed, providing indication of removal of the non-radiative carrier trapping states present in the bandgap of the NCs.

Figure 1(b-c) present transmission electron microscopy (TEM) images of CIGSe NCs. It is observed that the particles are spherical and the particle size varies from $~ 5.0-9.0 \mathrm{~nm}$. After $\mathrm{ZnS}$ shelling, the particles remain spherical (Figure 2(d-e)) and the particle size remains in the same range. Particle size distributions are provided in Figure S1 in the Supporting Information. It is well known that Zn-S treatment of multinary NCs leads to the formation of core-shell structures by exchange of the existing cations from the surface with $\mathrm{Zn}$ and that the particle size remains the same before and after shelling. ${ }^{39,}{ }^{69-70}$ However, Zn may diffuse as well inside the NCs to form an alloyed interfacial layer. ${ }^{29}, 39,70$ Inductively coupled plasma optical emission spectroscopy (ICP-OES) data indicate that the mole percentages of the cations in the NCs remain 48:28:24 for $\mathrm{Cu}: \mathrm{In}: \mathrm{Ga}$ while those for Se:S are 92:8. (S comes from the dodecanethiol used as the ligand). After $\mathrm{Zn}$ and $\mathrm{S}$ treatment, the mole percentages of $\mathrm{Cu}: \mathrm{In}: \mathrm{Ga}: \mathrm{Zn}$ are 37:25:20:18 and those for Se:S are 73:27. X-ray diffraction patterns of the CIGSe NCs before and after shelling show tetragonal structures (Supporting Information, Figure S2). 

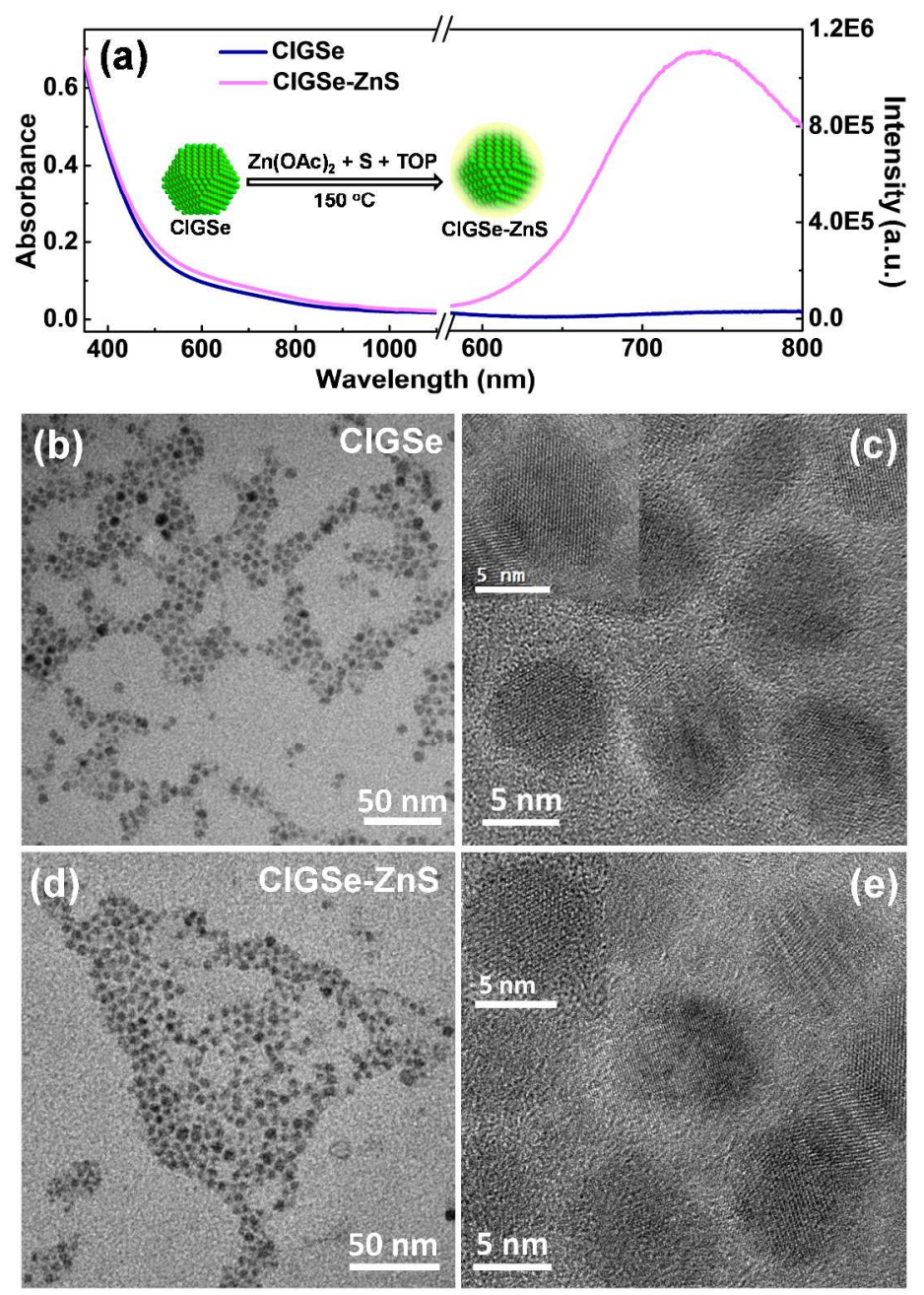

Figure 1. (a) The steady-state absorption (left) and emission spectra (right) of CIGSe NCs before and after $\mathrm{ZnS}$ shelling. The excitation wavelength for obtaining the emission spectra was $365 \mathrm{~nm}$ for both cases. (b-c) Representative TEM images of CIGSe NCs. (d-e) Representative TEM images of CIGSe-ZnS NCs. More HRTEM images have been provided in the supporting information (Figure S3).

To determine the charge carrier dynamics at the surface of the NCs, we took 4D S-UEM images of the thin films of CIGSe NCs before and after shelling. A schematic diagram of the 4D S-UEM setup is provided in Figure S4 of the Supporting Information; detailed information on the principle of its operation has been published elsewhere ${ }^{56,59}$ Briefly, the output of a fs Clark- 
MXR fiber laser system is integrated with a modified Quanta FEI-650 SEM. The IR pulses delivered by the laser are centered at $1030 \mathrm{~nm}$ with a pulse width of $\sim 270 \mathrm{fs}$ and the repetition rate ranging from $200 \mathrm{kHz}$ to $25.4 \mathrm{MHz}$. The fundamental output is split by a 1:1 beam splitter to pump two second/third harmonic generators (HG, Clark-MXR) simultaneously to produce 515 and $343 \mathrm{~nm}$ pulses. The $343 \mathrm{~nm}$ output is directed and tightly focused onto the emitter tip, which photo-generates electron pulses that are then accelerated using a $30 \mathrm{kV}$ voltage. The $515 \mathrm{~nm}$ output enters the microscope through a viewport that is at a 50-degree angle relative to the surface normal and is focused on the specimen to initiate the dynamics. The scanning process of the electron beam takes place across the surface of the sample, both the laser excited and unexcited regions in raster pattern, and the SEs emitted from the sample are detected by a positively biased Everhart-Thornley detector. The SE images are obtained as an integration of 64 frames with a dwell time of 300 ns at each pixel to improve the signal-to-noise ratio. Finally, all experiments are conducted at a repetition rate of $8 \mathrm{MHz}$ to ensure full recovery of the specimen before the arrival of the next pulse.

Figure 2 shows the time-resolved SE images obtained from the CIGSe NC thin film before and after shelling at different time delays after 515-nm excitation pulse. No change in the image contrast is observed at the far negative time delays ( $-327 \mathrm{ps})$, indicating the recovery of the system to the initial state after each pump-probe event. As can clearly be seen in both samples, a dark contrast evolves in the laser-illuminated region with time. The dark contrast at negative time can be attributed to the diffusion of plasmon-excited carriers that are generated from deeper areas of the sample by electron impact, which can be perturbed by electron-hole pairs generated by the clocking photon pulse, or to different scattering processes. ${ }^{58,62}$ At positive time delays, the 
optical pulse promotes a fraction of the valence-band electrons to the conduction band. Compared with electrons in an unexcited specimen, the promoted electrons have a higher probability of emitting SEs to the vacuum level when they are scattered by the energetic primary electron beam, which results in an enhanced probability of SE emission and should give rise to bright contrast in the laser illuminated region, as observed in case of $\mathrm{Si}$ or $\mathrm{CdSe} .{ }^{56}$ However, the dark contrast observed here suggests that SEs in the conduction band lose energy during transit to the surface and they do not reach the detector because of an energy deficit. ${ }^{58,61-62}$ In this case, scattering processes with photo-generated electron-hole pairs are most likely responsible for the energy loss. As the effective cross-section for the scattering of SEs with conduction electrons is much higher than that with valence electrons, ${ }^{58,61-62}$ a decrease in SE emission and, subsequently, low contrast are observed.

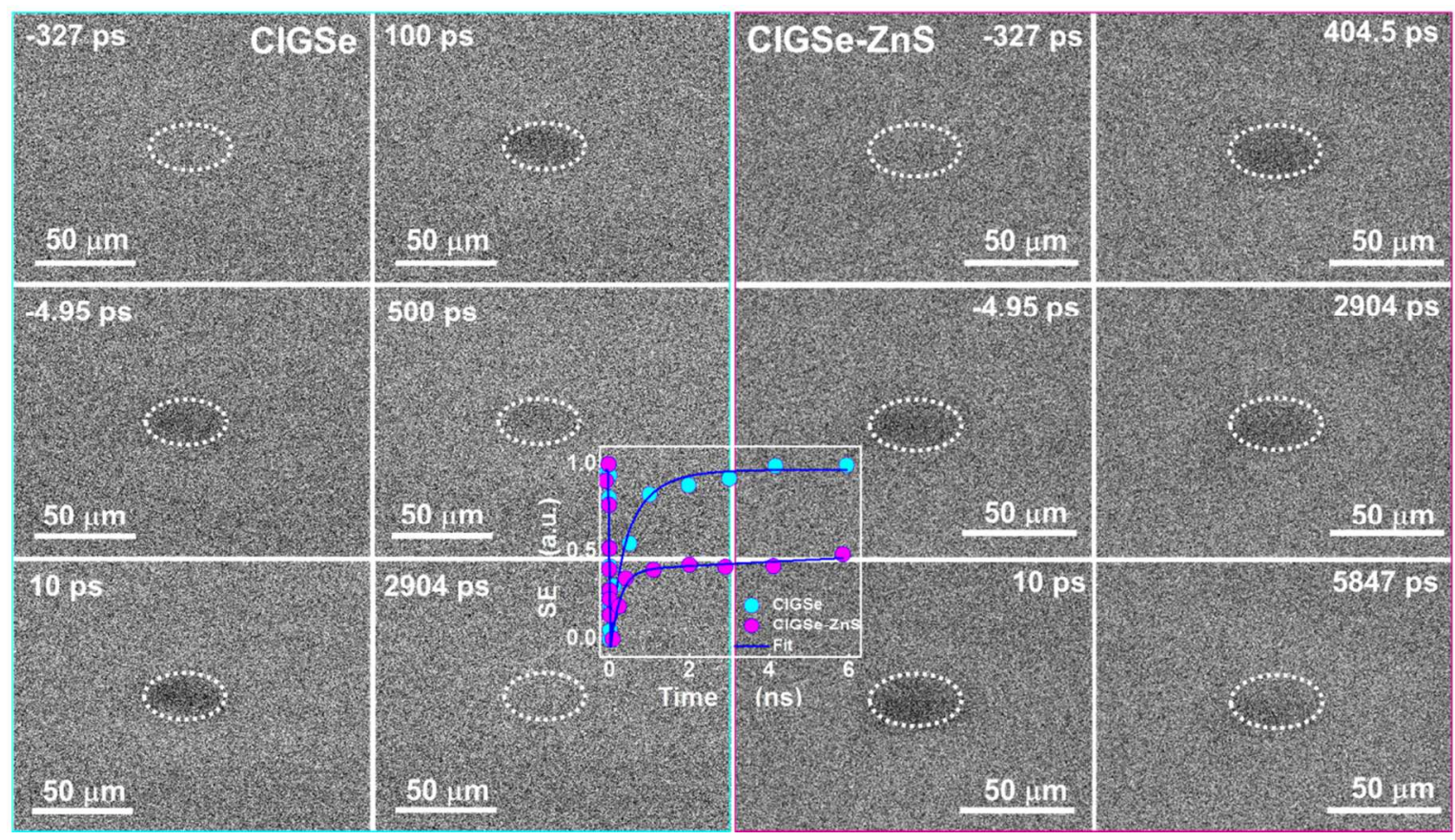

Figure 2. Time-resolved SE images from CIGSe and CIGSe-ZnS thin film at the indicated time delays. The dashed ellipses indicate the footprint of the clocking optical beam on the specimen 
$(\sim 40 \mu \mathrm{m})$. No observable change in the contrast at far negative time delays indicates the recovery of the system to an equilibrated state after each pump-probe event. The inset shows the dynamics of the temporal evolution of the SE intensity at the center of the laser footprint region. The SEM images for the thin films of the nanocrystals have been provided in the supporting information (Figure S5).

In spite of the SE signal being dark in both samples, it is clear that the signal fades away much faster for CIGSe than for CIGSe-ZnS, indicating fast carrier relaxation in the former. To extract the carrier dynamics, we plot the SE intensities from the center of the laser excitation footprint as function of the time delays between optical and electron pulses (inset, Figure 2). The pulse energy of the pump beam is $0.06 \mathrm{~nJ}$ at the sample. Within the time window of the experiment, the SE intensity fades away almost completely from CIGSe whereas only $40 \%$ of the signal fades away from CIGSe-ZnS.

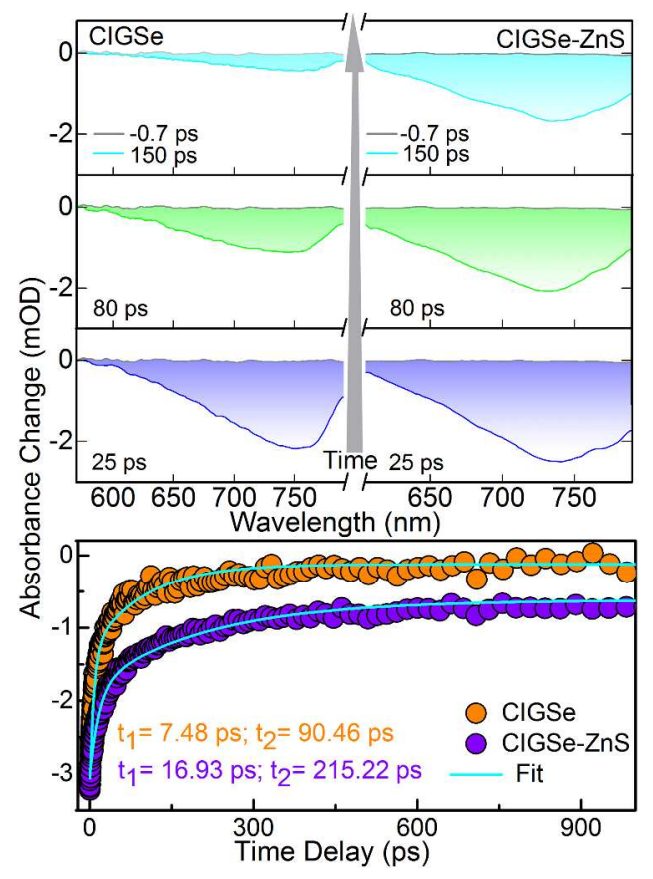

Figure 3. Top Panel: Transient absorption (TA) spectra of CIGSe and CIGSe-ZnS NCs at indicated delay time windows on the fs time scale following laser pulse excitation at $520 \mathrm{~nm}$. 
Bottom panel: Kinetics of the wavelength at the center of the corresponding ground-state bleach from the TA spectra of the NCs. The solid lines are fits for kinetic traces.

We further compared our imaging results with carrier dynamics obtained from ultrafast pumpprobe spectroscopy with fs and broadband capabilities for the NCs before and after Zn-S treatment. It is worth mentioning that a low pump fluence (the average number of photons absorbed per NC was less than 0.2 ) was used in all experiments. The probability of the formation of multi-exciton states through multi-photon absorption was therefore negligible. Figure 3 (top panel) shows the transient absorption (TA) spectra of the NCs on the fs time scale, obtained by probing with white light in the UV-Vis range, following $520 \mathrm{~nm}$ optical excitation. Clear ground-state bleach due to the removal of the occupied valence band population upon excitation is observed. The slower and smaller recovery of the ground-state bleach after $\mathrm{ZnS}$ shelling suggests minimization of the carrier relaxation pathways, which in turn provides clear evidence for significant reduction of the number of trap states after $\mathrm{ZnS}$ shelling. The shift in the groundstate bleach minima towards a lower wavelength suggests alloying with the $\mathrm{ZnS}$, a higher bandgap material. The kinetic traces of both the samples show one fast component in tens of picoseconds and another slow component in the range of hundreds of picoseconds (Figure 3, bottom panel), suggesting the respective presence of one shallow and one deep electron trap state. ${ }^{71}$ After $\mathrm{ZnS}$ shelling, the relative amplitude of the short-time component is reduced with respect to the long-time one, which suggests that a significant fraction of shallow electron traps have been removed. Also, the magnitudes of both time components increase, i.e., their positions move deeper with respect to the conduction band. However, the extent of the slowing down of the carrier dynamics after $\mathrm{ZnS}$ shelling is more discernible in the SE kinetics obtained by 4D SUEM. The reason for this remains the large penetration depth of the laser beam in ultrafast 
pump-probe spectroscopy, which acquires information from the bulk of the sample, whereas the SEs emitted from the first few nms of the top surface are detected by 4D S-UEM, which allows any dynamical changes in the surface to be more prominently recognized. We also measured the TA spectra of the CIGSe-ZnS NCs in the nanosecond time scale following $520 \mathrm{~nm}$ optical excitation. The kinetics shows two time components of $5.11 \mathrm{~ns}$ and $152.11 \mathrm{~ns}$ (details provided in Supporting Information, Figure S6), both of which come from radiative recombination, as confirmed from time-correlated single photon counting (TCSPC) measurements. However, this measurement cannot be performed for CIGSe NCs because of their extremely short lifetime. Significant elongation of charge carrier dynamics and radiative lifetime clearly demonstrate the effectiveness of $\mathrm{ZnS}$ shelling in passivating the non-radiative surface trap states of the NCs.

(a)

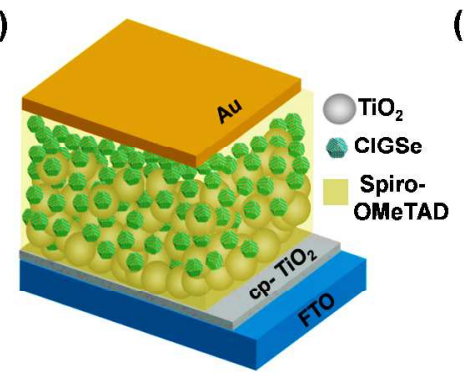

(c)

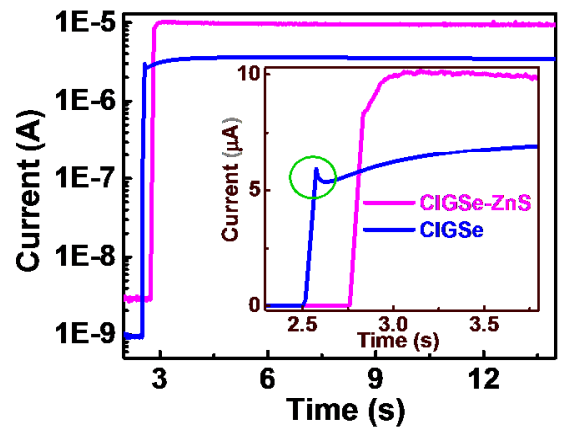

(b)

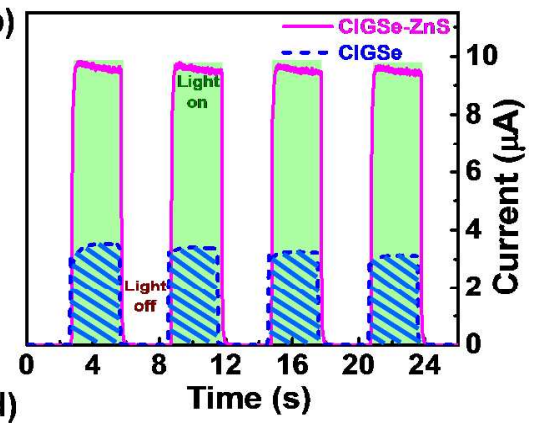

(d)

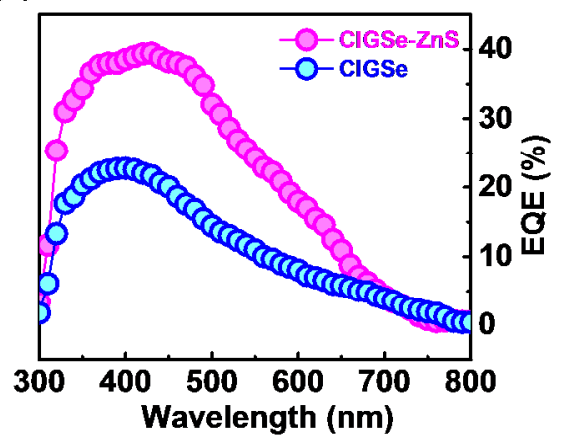

Figure 4. (a) Schematic of the CIGSe NC sensitized photodetector. (b-c) Current response vs time under (b) chopped and (c) continuous irradiation (bias voltage of $0 \mathrm{~V}$, wavelength $510 \mathrm{~nm}$, $10 \mathrm{~mW} / \mathrm{cm}^{2}$ ). The inset in (c) shows a magnified version of the spectra in the first few seconds. 
The encircled area shows fast photocurrent decay of the CIGSe-based device during the initial illumination. (d) The spectral dependence of the Se and CIGSe-ZnS photodetectors (measured using external quantum efficiencies at zero bias).

As the charge carrier lifetime of any material has a direct impact on its light-harvesting properties, we compared the self-biased photo-detection performance of CIGSe NCs before and after shelling. We opted for a simple solution-processed, mesoscopic quantum-dot-sensitized design to reduce fabrication costs. The experimental details are given in the supporting information. Figure 4(a) shows a schematic of our fabricated mesoscopic quantum-dot-sensitized self-biased photodetector. The self-biased photo-detection performance was measured by illuminating the device under chopped/continuous illumination of wavelength $510 \mathrm{~nm}$ and intensity of 0.10 Sun $\left(10 \mathrm{~mW} / \mathrm{cm}^{2}\right)$ at zero applied bias. Figure $4(\mathrm{~b}-\mathrm{c})$ shows the time response of the photocurrent for the chopped illumination (3 sec on, $3 \mathrm{sec}$ off) and the continuous illumination of light. Both figures show that, for the CIGSe NCs, soon after light illumination (less than the instrumental limit of $5 \mathrm{~ms}$ ), the photocurrent value increases about four orders of magnitude higher than the dark current value. Once the illumination is off, it immediately comes back to its dark value indicating that our device is a highly efficient self-biased photodetector with an on/off ratio of the order of $10^{4}$ and a fast response time of less than $5 \mathrm{~ms}$. The photoresponsivity was obtained from the ratio of the photocurrent $\left(\Delta I=J_{\text {Photo }}-J_{\text {Dark }}\right)$ to the incident light intensity $\left(\mathrm{R}=\Delta I / L_{\text {Light }}\right.$, where $\mathrm{L}_{\text {Light }}$ is the intensity of the incident light $){ }^{72}$ The estimated photo-responsivity for the CIGSe-ZnS based photodetector is $0.25 \mathrm{~A} / \mathrm{W}$, which is three times higher than that of the CIGSe based device. This is most likely due the sharp decrease in the photocurrent within the first few seconds after illumination in the CIGSe device as indicated in Figure 4(c) (inset, encircled area). This trend of photocurrent decay in the initial seconds of 
illumination can be seen during repeated on-off cycles of illumination (encircled areas in Figure S7). No such decay in the photocurrent was observed with the CIGSe-ZnS device and the nature of the photocurrent was determined to be similar to typical photoelectrochemical cells. ${ }^{73}$ It has been proposed in literature that the observed photocurrent decay during the initial excitation period may arise from: (i) the inability of the hole-transporting materials to quickly scavenge photo-generated holes or (ii) dominant charge recombination/scattering at the $\mathrm{NC} / \mathrm{TiO}_{2}$ heterointerface or at grain boundaries within the mesoporous $\mathrm{TiO}_{2}{ }^{74}$ As the hole-transporting material in both of the devices remains same, the sharp decay of photocurrent in the CIGSe case suggests the presence of a large number of trap states in the CIGSe NCs, which promote photocarrier recombination at the $\mathrm{CIGSe} / \mathrm{TiO}_{2}$ interface. The slow increase in the photocurrent value after this sharp decay is most likely the signature of trap filling after a sufficient illumination time. Absence of a similar decay for CIGSe-ZnS device supports efficient removal of the carrier trapping states. To confirm the self-biasing behavior of our photo-detectors, we also measured the photovoltaic performance of both the devices. Almost two times higher efficiency of the CIGSe-ZnS device further substantiates the efficacy of $\mathrm{ZnS}$ shelling removing the non-radiative carrier trapping channels (details provided in Supporting Information, Figure S8). Further, the wavelength dependency of the photodetectors was studied by measuring the external quantum efficiency (EQE) of the CIGSe and CIGSe-ZnS devices by monitoring the photocurrent at different wavelengths at zero bias (Figure 4(d)). The photocurrent value starts around $750 \mathrm{~nm}$ for both devices with a small blue shift for the CIGSe-ZnS device. The EQE value is remarkably higher for the CIGSe-ZnS device compared to that of the CIGSe one, indicating its higher efficiency. 
To conclude, the ability to probe carrier dynamics on material surfaces and interfaces is a particularly challenging task. Here, we employ 4D S-UEM with sub-picosecond temporal resolution and nanometer spatial resolution to directly probe in time and space the effect of shelling ZnS on the surface charge carrier dynamics of CIGSe NCs, which is beyond the capabilities of available time-resolved laser spectroscopic techniques and static electron microscopy. The time-resolved SE snapshots clearly demonstrate that the density of the trap states is significantly reduced after shelling with $\mathrm{ZnS}$. In addition, our data indicates that the presence of trap states has a crucial effect on device performance; and hence almost three times higher photocurrent value is obtained from the nanocrystals after shelling. A clear signature of trap state removal is also evident in the time-resolved photocurrent of the CIGSe-ZnS NCs. Our study not only provides fundamental insights about carrier dynamics selectively on nanoscalematerial surfaces but also leads the way to design effective passivation strategies for NCs to enhance their performance in photoactive applications.

\section{Experimental Section}

\section{Synthesis of nanocrystals:}

Materials: Copper (I) chloride ( $\mathrm{CuCl}, 99.99 \%)$, Indium (III) chloride ( $\mathrm{InCl}_{3}, 98 \%$ ), Gallium (III) chloride $\left(\mathrm{GaCl}_{3}, 99.99 \%\right)$, Zinc (II) acetate $\left(\mathrm{Zn}(\mathrm{OAc})_{2}\right.$, tech.), 1-Dodecanethiol (DDT, >98\%), Octadecylamine (ODA, 97\%), Oleylamine (OAm, tech.), Octadecene (ODE, tech.), Sulfur powder (S), 3-mercaptopropyl trimethoxysilane (MPTMS), ethanedithiol (EDT) were purchased from Aldrich. Se powder (200 mesh, 99.99\%) was purchased from Alfa-Aesar. All the chemicals were used as received without further purification.

Methods: 
Preparation of $\mathbf{C u} / \mathbf{I n} / \mathbf{G a}$ stock solution: The $\mathrm{Cu}$, In and $\mathrm{Ga}$ stock solutions were prepared by dissolving $0.5 \mathrm{mmol}$ of $\mathrm{CuCl}(0.049 \mathrm{~g}), 0.25 \mathrm{mmol}$ of $\mathrm{InCl}_{3}(0.055 \mathrm{~g}), 0.25 \mathrm{mmol}$ of $\mathrm{GaCl}_{3}$ in 2.5 mL OAm each in three separate vials. The vials were degassed by purging them with $\mathrm{N}_{2}$.

Preparation of Se stock solution: The Se stock solution was prepared by dissolving $1 \mathrm{mmol}$ of Se powder $(0.079 \mathrm{~g})$ in $0.5 \mathrm{~mL}$ DDT and $0.5 \mathrm{~mL}$ OAm. The vial was degassed by purging them with $\mathrm{N}_{2}$.

Synthesis of CIGSe nanorcystals: In a typical procedure, $7 \mathrm{~mL}$ ODE was taken in a three-neck flask and degassed by purging with $\mathrm{N}_{2}$ for $15 \mathrm{~min}$. In a separate vial, $0.5 \mathrm{~mL}$ of each cation solution was dissolved in $0.5 \mathrm{~mL}$ DDT and $0.5 \mathrm{~mL}$ OAm, degassed and heated until a clear yellow solution was formed. Then, the flask was heated to $200{ }^{\circ} \mathrm{C}$ and $0.2 \mathrm{~mL}$ Se stock solution and the cation solution was injected back into the flask. The color of the solution darkened immediately, indicating the formation of NCs. The reaction mixture was annealed for $10 \mathrm{~min}$. Then, the reaction mixture was cooled down and the synthesized NCs were purified using ethanol as well as acetone as non-solvents and chloroform as a dispersing solvent.

Shelling of CIGSe nanocrystals with ZnS: For shelling with ZnS, a modified method from the literature was followed. ${ }^{24}$ In a typical procedure, a stock solution of $0.05 \mathrm{mmol} \mathrm{Zn}(\mathrm{OAc})_{2}$ $(0.009 \mathrm{~g})$ and $0.05 \mathrm{mmol} \mathrm{S}$ powder $(0.0016 \mathrm{~g})$ dissolved in $0.5 \mathrm{~mL}$ ODE and $0.2 \mathrm{~mL}$ TOP. This solution was drop-wise added at $150{ }^{\circ} \mathrm{C}$ to the purified $\mathrm{NCs}$ dispersed in $0.5 \mathrm{~g}$ ODA and $8 \mathrm{~mL}$ of ODE. The reaction was then annealed for $10 \mathrm{~min}$. Finally, the reaction mixture was cooled to room temperature, and the NCs were purified using ethanol as well as acetone as nonsolvents and chloroform as a dispersing solvent. 


\section{ASSOCIATED CONTENT}

Supporting Information. Instrumentation, details of device fabrication procedure and supporting figures. This material is available free of charge via the Internet at http://pubs.acs.org

\section{AUTHOR INFORMATION}

\section{Corresponding Author}

*Email. omar.abdelsaboor@kaust.edu.sa

\section{REFERENCES}

1. Carey, G. H.; Abdelhady, A. L.; Ning, Z.; Thon, S. M.; Bakr, O. M.; Sargent, E. H., Chem. Rev. 2015, 115, 12732-12763.

2. Kamat, P. V., Quantum Dot Solar Cells. J. Phys. Chem. Lett. 2013, 4, 908-918.

3. Banin, U., Light-emitting diodes. Nat. Photonics 2008, 2, 209-210.

4. Anikeeva, P. O.; Halpert, J. E.; Bawendi, M. G.; Bulovic, V., Nano Lett. 2009, 9, 25322536.

5. Konstantatos, G.; Howard, I.; Fischer, A.; Hoogland, S.; Clifford, J.; Klem, E.; Levina, L.; Sargent, E. H., Nature 2006, 442, 180-183.

6. Sukhovatkin, V.; Hinds, S.; Brzozowski, L.; Sargent, E. H., Science 2009, 324, 15421544.

7. Bekenstein, Y.; Koscher, B. A.; Eaton, S. W.; Yang, P.; Alivisatos, A. P., J. Am. Chem. Soc. 2015, 137, 16008-16011. 
8. Kovalenko, M. V.; Manna, L.; Cabot, A.; Hens, Z.; Talapin, D. V.; Kagan, C. R.; Klimov, V. I.; Rogach, A. L.; Reiss, P.; Milliron, D. J.; Guyot-Sionnnest, P.; Konstantatos, G.; Parak, W. J.; Hyeon, T.; Korgel, B. A.; Murray, C. B.; Heiss, W., ACS Nano 2015, 9, 1012-1057.

9. Rogach, A. L.; Gaponik, N.; Lupton, J. M.; Bertoni, C.; Gallardo, D. E.; Dunn, S.; Li Pira, N.; Paderi, M.; Repetto, P.; Romanov, S. G.; O'Dwyer, C.; Sotomayor Torres, C. M.; Eychmüller, A., Angew. Chem. Int. Ed. 2008, 47, 6538-6549.

10. Steckel, J. S.; Snee, P.; Coe-Sullivan, S.; Zimmer, J. P.; Halpert, J. E.; Anikeeva, P.; Kim, L.-A.; Bulovic, V.; Bawendi, M. G., Angew. Chem. Int. Ed. 2006, 45, 5796-5799.

11. Morello, G.; Anni, M.; Cozzoli, P. D.; Manna, L.; Cingolani, R.; De Giorgi, M., J. Phys. Chem. C 2007, 111, 10541-10545.

12. Klimov, V. I.; McBranch, D. W.; Leatherdale, C. A.; Bawendi, M. G., Electron and hole relaxation pathways in semiconductor quantum dots. Phys. Rev. B: Condens. Matter Mater. Phys. 1999, 60, 13740-13749.

13. Mooney, J.; Krause, M. M.; Saari, J. I.; Kambhampati, P., Phys. Rev. B 2013, 87, 081201.

14. Jones, M.; Lo, S. S.; Scholes, G. D., Proc. Natl. Acad. Sci. 2009, 106, 3011-3016.

15. Boles, M. A.; Ling, D.; Hyeon, T.; Talapin, D. V., Nat Mater 2016, 15, 141-153.

16. Panthani, M. G.; Akhavan, V.; Goodfellow, B.; Schmidtke, J. P.; Dunn, L.; Dodabalapur, A.; Barbara, P. F.; Korgel, B. A., J. Am. Chem. Soc. 2008, 130, 16770-16777.

17. Steinhagen, C.; Panthani, M. G.; Akhavan, V.; Goodfellow, B.; Koo, B.; Korgel, B. A., J. Am. Chem. Soc. 2009, 131, 12554-12555. 
18. McLeod, S. M.; Hages, C. J.; Carter, N. J.; Agrawal, R., Synthesis and characterization of 15\% efficient CIGSSe solar cells from nanoparticle inks. Prog. Photovol. 2015, 23, 1550-1556.

19. Zhao, X.; Lu, M.; Koeper, M.; Agrawal, R., J. Mater. Chem. A 2016, DOI: 10.1039/C6TA00533K.

20. Chen, L.-J.; Liao, J.-D.; Chuang, Y.-J.; Fu, Y.-S., J. Am. Chem. Soc. 2011, 133, 37043707.

21. Ford, G. M.; Guo, Q.; Agrawal, R.; Hillhouse, H. W., Chem. Mater. 2011, 23, 26262629.

22. Jackson, P.; Hariskos, D.; Wuerz, R.; Kiowski, O.; Bauer, A.; Friedlmeier, T. M.; Powalla, M., phys. status solidi-Rapid Res. Lett. 2015, 9, 28-31.

23. Aldakov, D.; Lefrancois, A.; Reiss, P., J. Mater. Chem. C 2013, 1, 3756-3776.

24. Li, L.; Pandey, A.; Werder, D. J.; Khanal, B. P.; Pietryga, J. M.; Klimov, V. I., J. Am. Chem. Soc. 2011, 133, 1176-1179.

25. Castro, S. L.; Bailey, S. G.; Raffaelle, R. P.; Banger, K. K.; Hepp, A. F., J. Phys.Chem. B 2004, 108, 12429-12435.

26. Tang, J.; Hinds, S.; Kelley, S. O.; Sargent, E. H., Chem. Mater. 2008, 20, 6906-6910.

27. Li, L.; Daou, T. J.; Texier, I.; Kim Chi, T. T.; Liem, N. Q.; Reiss, P., Chem. Mater. 2009, $21,2422-2429$.

28. Pons, T.; Pic, E.; Lequeux, N.; Cassette, E.; Bezdetnaya, L.; Guillemin, F.; Marchal, F.; Dubertret, B., ACS Nano 2010, 4, 2531-2538. 
29. Nam, D.-E.; Song, W.-S.; Yang, H., Facile, J. Mater. Chem. 2011, 21, 18220-18226.

30. Ip, A. H.; Thon, S. M.; Hoogland, S.; Voznyy, O.; Zhitomirsky, D.; Debnath, R.; Levina, L.; Rollny, L. R.; Carey, G. H.; Fischer, A.; Kemp, K. W.; Kramer, I. J.; Ning, Z.; Labelle, A. J.; Chou, K. W.; Amassian, A.; Sargent, E. H., Nat Nano 2012, 7, 577-582.

31. Tang, J.; Huo, Z.; Brittman, S.; Gao, H.; Yang, P., Nat Nano 2011, 6, 568-572.

32. Jun, S.; Jang, E., Bright and Stable Alloy Core/Multishell Quantum Dots. Angew. Chem. Int. Ed. 2013, 52, 679-682.

33. Jung, J.; Lin, C. H.; Yoon, Y. J.; Malak, S. T.; Zhai, Y.; Thomas, E. L.; Vardeny, V.; Tsukruk, V. V.; Lin, Z., Angew. Chem. Inter. Ed. 2016, DOI: 10.1002/anie.201601198.

34. Cao, Y.-W.; Banin, U., Angew. Chem., Int. Ed. 1999, 38, 3692-3694.

35. Völker, J.; Zhou, X.; Ma, X.; Flessau, S.; Lin, H.; Schmittel, M.; Mews, A., Angew. Chem. Int. Ed. 2010, 49, 6865-6868.

36. Zhang, J. Z., Acc. Chem. Res. 1997, 30, 423-429.

37. Chuang, C.-H.; Burda, C., J. Phys. Chem. Lett. 2012, 3, 1921-1927.

38. Burda, C.; Link, S.; Mohamed, M.; El-Sayed, M., J. Phys. Chem. B 2001, 105, 1228612292.

39. Bose, R.; Ahmed, G. H.; Alarousu, E.; Parida, M. R.; Abdelhady, A. L.; Bakr, O. M.; Mohammed, O. F., J. Phys. Chem. C 2015, 119, 3439-3446.

40. Wan, Y.; Guo, Z.; Zhu, T.; Yan, S.; Johnson, J.; Huang, L., Nat Chem 2015, 7, 785-792. 
41. Guo, Z.; Manser, J. S.; Wan, Y.; Kamat, P. V.; Huang, L., Nat Commun 2015, 6.

42. Gabriel, M. M.; Kirschbrown, J. R.; Christesen, J. D.; Pinion, C. W.; Zigler, D. F.; Grumstrup, E. M.; Mehl, B. P.; Cating, E. E. M.; Cahoon, J. F.; Papanikolas, J. M., Nano Lett. 2013, 13, 1336-1340.

43. Kwon, O.-H.; Barwick, B.; Park, H. S.; Baskin, J. S.; Zewail, A. H., Nano Lett. 2008, 8, 3557-3562.

44. Kwon, O.-H.; Park, H. S.; Baskin, J. S.; Zewail, A. H., Nano Lett. 2010, 10, 3190-3198.

45. Liu, H.; Kwon, O.-H.; Tang, J.; Zewail, A. H., Nano Lett. 2014, 14, 946-954.

46. Carbone, F.; Kwon, O.-H.; Zewail, A. H., Science 2009, 325, 181-184.

47. Flannigan, D. J.; Park, S. T.; Zewail, A. H., Nano Lett. 2010, 10, 4767-4773.

48. Flannigan, D. J.; Samartzis, P. C.; Yurtsever, A.; Zewail, A. H., Nano Lett. 2009, 9, 875881.

49. Flannigan, D. J.; Zewail, A. H., Nano Lett. 2010, 10, 1892-1899.

50. Flannigan, D. J.; Zewail, A. H., 2012, 45, 1828-1839.

51. Zewail, A. H., Science 2010, 328, 187-193.

52. Najafi, E.; Scarborough, T. D.; Tang, J.; Zewail, A., Science 2015, 347, 164-167.

53. Lobastov, V. A.; Srinivasan, R.; Zewail, A. H., Proc. Natl. Acad. Sci 2005, 102, 70697073.

54. Park, H. S.; Baskin, J. S.; Kwon, O.-H.; Zewail, A. H., Nano Lett. 2007, 7, 2545-2551. 
55. Park, H. S.; Baskin, J. S.; Zewail, A. H., Nano Lett. 2010, 10, 3796-3803.

56. Mohammed, O. F.; Yang, D.-S.; Pal, S. K.; Zewail, A. H., J. Am. Chem. Soc. 2011, 133, 7708-7711.

57. Yang, D.-S.; Mohammed, O. F.; Zewail, A. H., Proc. Natl. Acad. Sci. 2010, 107, 1499314998.

58. Cho, J.; Hwang, T. Y.; Zewail, A. H., Proc. Nal. Acad. Sci. 2014, 111, 2094-2099.

59. Sun, J.; Melnikov, V. A.; Khan, J. I.; Mohammed, O. F., J. Phys. Chem. Lett. 2015, 6, 3884-3890.

60. Sun, J.; Adhikari, A.; Shaheen, B. S.; Yang, H.; Mohammed, O. F., J. Phys. Chem. Lett. 2016, 7, 985-994.

61. Khan, J. I.; Adhikari, A.; Sun, J.; Priante, D.; Bose, R.; Shaheen, B. S.; Ng, T. K.; Zhao, C.; Bakr, O. M.; Ooi, B. S.; Mohammed, O. F., Small 2016, DOI: 10.1002/smll.201503651.

62. Bose, R.; Sun, J.; Khan, J. I.; Shaheen, B. S.; Adhikari, A.; Ng, T. K.; Burlakov, V. M.; Parida, M. R.; Davide, P.; Goriely, A.; Ooi, B. S.; Bakr, O. M.; Mohammed, O. F., Adv. Mater. 2016, DOI: $10.1002 / a d m a .201600202$.

63. Reimer, L., Scanning Electron Microscopy. 2 ed.; Springer: Vol. 45.

64. Wang, Y.-H. A.; Zhang, X.; Bao, N.; Lin, B.; Gupta, A., J. Am. Chem. Soc. 2011, 133, 11072-11075.

65. Zhong, H.; Wang, Z.; Bovero, E.; Lu, Z.; van Veggel, F. C. J. M.; Scholes, G. D., J. Phys. Chem. C 2011, 115, 12396-12402. 
66. Liu, Y.; Yao, D.; Shen, L.; Zhang, H.; Zhang, X.; Yang, B., J. Am. Chem. Soc. 2012, 134, 7207-7210.

67. Song, W.-S.; Kim, J.-H.; Lee, J.-H.; Lee, H.-S.; Do, Y. R.; Yang, H., J. Mater. Chem 2012, 22, 21901-21908.

68. Uematsu, T.; Doi, T.; Torimoto, T.; Kuwabata, S., J. Phys. Chem. Lett. 2010, 1, 32833287.

69. Pan, Z.; Mora-Seró, I.; Shen, Q.; Zhang, H.; Li, Y.; Zhao, K.; Wang, J.; Zhong, X.; Bisquert, J., J. Am. Chem. Soc. 2014, 136, 9203-9210.

70. Park, J.; Kim, S.-W., J. Mater. Chem. 2011, 21, 3745-3750.

71. Sun, J.; Yang, Y.; Khan, J. I.; Alarousu, E.; Guo, Z.; Zhang, X.; Zhang, Q.; Mohammed, O. F., ACS Appl. Mater. Interfaces 2014, 6, 10022-10027.

72. Gong, X.; Tong, M.; Xia, Y.; Cai, W.; Moon, J. S.; Cao, Y.; Yu, G.; Shieh, C.-L.; Nilsson, B.; Heeger, A. J., Science 2009, 325, 1665-1667.

73. Christians, J. A.; Fung, R. C. M.; Kamat, P. V., J. Am. Chem. Soc. 2014, 136, 758-764.

74. Robel, I.; Subramanian, V.; Kuno, M.; Kamat, P. V., J. Am. Chem. Soc. 2006, 128, 23852393. 
Table of Contents Graphic

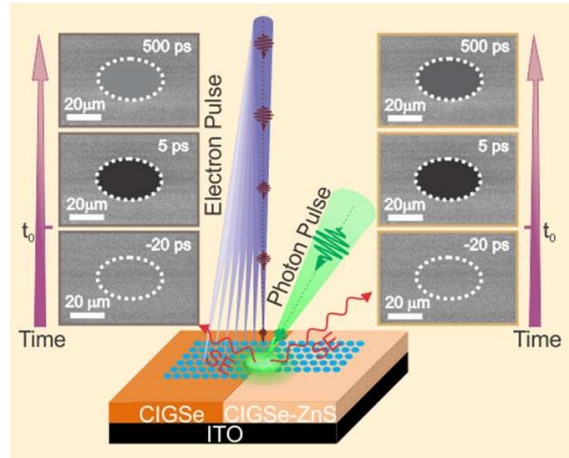

2

4

5

6

10

11

12

13

14

15

16

17

18

19

20

21

22

23

24

25

26

27

28

29

30

31

32

33

34

35

36

37

38

39

40

41

42

43

44

45

46

47

48

49

50

51

52

53

54

55

56

57

58

59

60 